\title{
Diffusion and anomalous diffusion of light in two-dimensional photonic crystals
}

\author{
A. A. Asatryan, ${ }^{1,2}$ P. A. Robinson, ${ }^{2}$ R. C. McPhedran, ${ }^{2}$ L. C. Botten,${ }^{1}$ C. Martijn de Sterke, ${ }^{2}$ T. L. Langtry, ${ }^{1}$ N. A. \\ Nicorovici $^{2}$ \\ ${ }^{1}$ School of Mathematical Sciences, University of Technology, Sydney, N.S.W. 2007, Australia \\ ${ }^{2}$ School of Physics, University of Sydney, NSW 2006, Australia
}

\begin{abstract}
The transport properties of electromagnetic waves in disordered, finite, two-dimensional photonic crystals composed of circular cylinders are considered. Transport parameters, such as the transport and scattering mean free paths and the transport velocity are calculated, for the case where the electromagnetic radiation has its electric field along the cylinder axes. The range of the parameters in which the diffusion process can take place is specified. It is shown that the transport velocity $v_{E}$ can be as much as $10^{8}$ times less than its free space value, while just outside the cluster $v_{E}$ can be $0.3 c$. The effects of weak and strong disorder on the transport velocity are investigated. The different regimes of the wave transport: ordered propagation, diffusion and anomalous diffusion are demonstrated and it is inferred that Anderson localization is incipient in the latter regime. Exact numerical calculations from the Helmholtz equation are shown to be in good agreement with the diffusion approximation.
\end{abstract}

\section{INTRODUCTION}

Propagation of electromagnetic waves in ordered and disordered media has attracted much interest recently [1], partly born of the invention of photonic crystals [2,3] -materials with a periodic refractive index distribution. Such materials can prohibit propagation of light in all directions and for all polarizations at so-called gap wavelengths $[4,5]$. Therefore a photon with a gap wavelength is trapped or localized inside such structure and we term this gap localization.

In contrast to gap localization, Anderson localization of photons, the analog of electron Anderson localization, takes place in a disordered medium. Due to multiple scattering of photons off the equivalent random potential created by variability in optical properties such as refractive index, wave transport undergoes a phase transition from propagation to exponential decay [6]. To demonstrate Anderson localization of photons, John [2] suggested use of disordered, but periodic on average, photonic crystals composed of dielectric inclusions in a matrix [4,5].

One of the crucial parameters of Anderson localization is the linear "sample size" $L$ of the random medium. As waves propagate inside the random sample the original coherent propagation changes to diffusive propagation with a scattered transport mean free path $l_{t}$. Interference of waves can then reduce the transport mean free path $l_{t}$ and renormalize the diffusion coefficient $D$, to a form that depends on the sample size and disorder. Such renormalization of the diffusion coefficient leads to anomalous diffusion, and then to localization if $D \rightarrow 0$. The scaling theory of localization [7] predicts that, in an infinite medium, waves with all wavelengths are localized in one and twodimensional infinite media even for a small amount of disorder, while for three dimensional problems there exists a region of wavelengths in which the localization takes place at a given level of disorder. However, all realistic random samples are finite. Therefore it is important to take into account the effects of the finite size of the random samples on localization properties. Furthermore the finiteness of the sample allows the possibility of a diffusive regime of wave propagation even for one and two dimensional problems.

Since the suggestion by John [2] to use randomized photonic crystals to demonstrate Anderson localization of photons, only a few papers have considered this and related problems. Some aspects of the localization of waves in general have been considered [8], particularly for two-dimensional problems [9-11].

The diffusion coefficient $D$ is closely related to the transport velocity of light $v_{E}$, which has been calculated in the limit of low concentration of scatterers [12,13]. The question of the transport velocity of light $v_{E}$ for high concentrations of scatterers has been a difficult problem so far [14]. Effective medium models based on the spectral function approach $[15]$ and the approach of averaged energy density homogeneity $[16,17]$ have been developed to model this problem, but their limits of applicability are restricted $[18,19]$.

A recent exact multipole expansion method [20-25] and the construction of the Green tensor $G_{i j}\left(\mathbf{r}_{\mathbf{1}}, \mathbf{r}_{\mathbf{s}}\right)[26,27]$ allows one to calculate the transport velocity $v_{E}$ for high concentration of scatterers with high accuracy for two dimensional problems. The method also allows one to investigate the transport properties of random media such as the diffusion coefficient $D$ and the correlation function $\left\langle G_{i}\left(\mathbf{r}_{1}, \mathbf{r}_{\mathbf{s}}\right) G_{j}^{*}\left(\mathbf{r}_{\mathbf{2}}, \mathbf{r}_{\mathbf{s}}\right)\right\rangle$ [14].

In general one can specify four regimes of wave propagation in disordered media: The homogenized regime, in which the random medium can be characterized by an effective dielectric constant $\varepsilon_{\text {eff }}$, applies when the wavelength 
is greater than that of any characteristic structures within the medium ; i.e., $\lambda \gg L_{s}$, where $L_{s}$ is the size of the scatterers or the distance between them. The diffusive regime, in which the intensity of the waves obeys the diffusion equation, occurs when $\lambda / 2 \pi \ll l_{t} \ll L$ holds, where $l_{t}$ is the transport mean free path and $L$ is the size of the sample [28]. The regime in which the transport mean free path is a function of the size of the cluster and/or the degree of disorder, called the anomalous diffusive regime. Finally, in the localized regime we have $\lambda / 2 \pi \gtrsim l_{t}$, which is known as the Ioffe-Regel criterion.

Here we investigate the different regimes of wave propagation: diffusion, anomalous diffusion and Anderson localization for both pass-band and gap wavelengths. Other aims of this paper are to calculate rigorously the transport velocity $v_{E}$ of light in finite sized photonic crystals for intermediate concentrations of scatterers where no theoretical models exist, and to investigate the effects of disorder on $v_{E}$.

In Sec. II we briefly outline the construction of the Green function. In Sec. III we calculate the transport and scattering mean free paths, $l_{t}$ and $l_{s}$, of photons and determine the values of these parameters where diffusive propagation of waves can take place. In Sec. IV we calculate the transport velocity $v_{E}$ of waves inside finite photonic crystals from first principles. In Sec. $\mathrm{V}$ we show that light propagation in disordered photonic crystals of finite size can be diffusive and also demonstrate the anomalous regime of wave propagation. Incipient Anderson localization for gap wavelengths is also inferred.

\section{GREEN FUNCTION}

In the recent paper [27] we constructed the Green tensor for two dimensional photonic crystals composed of a finite cluster of $N_{c}$ non-overlapping circular inclusions in a matrix, as illustrated in Fig.1. The radii of inclusions in a matrix $a_{l}$, refractive indices $n_{l}$, and positions $\mathbf{c}_{l}$ of the circle centers are otherwise arbitrary. A point source is assumed to be located at $\mathbf{c}_{s}$. This system can also be viewed as representing a collection of parallel circular cylinders with the propagation strictly perpendicular to their axes from a line source and we will use the two descriptions interchangeably below.

Here we give a brief outline of the method that we use to calculate the component of the Green tensor for $E_{z}$ polarization in the subsequent sections. For this polarization the electric vector is aligned along the axes of the cylinders and the electromagnetic field can be described by a single component of the electric Green tensor $G_{z z}=V^{e}\left(\mathbf{r} ; \mathbf{c}_{s}\right)$ that obeys the Helmholtz equation

$$
\nabla^{2} V^{e}\left(\mathbf{r} ; \mathbf{c}_{s}\right)+k^{2} n^{2}(\mathbf{r}) V^{e}\left(\mathbf{r} ; \mathbf{c}_{s}\right)=\delta\left(\mathbf{r}-\mathbf{c}_{s}\right),
$$

where $V^{e}$ and $\nu \cdot \nabla V^{e}$ are continuous across all cylinder boundaries. Here $n(\mathbf{r})$ is the refractive index of the cylinders or the matrix and $\nu$ is the local outward unit vector normal to the surface of the cylinders.

To construct the Green function we use exact multipole expansions in which the wave fields inside and outside the cylinders are expressed in terms of Bessel functions with unknown coefficients. By using the boundary conditions of the continuity of the field and its derivative at the surfaces of the cylinders and by applying Green's second theorem over the cluster we derive a linear set of equations for the unknown coefficients [26]. By solving the linear set of equations, the Green function is then reconstructed as

$$
\begin{aligned}
V^{e}\left(\mathbf{r} ; \mathbf{c}_{s}\right) & =\chi^{\operatorname{ext}}\left(\mathbf{c}_{s}\right) H_{0}^{(1)}\left(k\left|\mathbf{r}-\mathbf{c}_{s}\right|\right) /(4 i) \\
& +\sum_{q=1}^{N_{c}} \sum_{m=-\infty}^{\infty} B_{m}^{q} H_{m}^{(1)}\left(k\left|\mathbf{r}-\mathbf{c}_{q}\right|\right) e^{i m \arg \left(\mathbf{r}-\mathbf{c}_{q}\right)}
\end{aligned}
$$

for points in the matrix, and

$$
\begin{aligned}
V^{e}\left(\mathbf{r} ; \mathbf{c}_{s}\right) & =\chi_{l}^{\mathrm{int}}\left(\mathbf{c}_{s}\right) H_{0}^{(1)}\left(k n_{l}\left|\mathbf{r}-\mathbf{c}_{s}\right|\right) /(4 i) \\
& +\sum_{m=-\infty}^{\infty} C_{m}^{l} J_{m}\left(k n_{l}\left|\mathbf{r}-\mathbf{c}_{l}\right|\right) e^{i m \arg \left(\mathbf{r}-\mathbf{c}_{l}\right)}
\end{aligned}
$$

for points inside the cylinders. The term $\chi^{\operatorname{ext}}\left(\mathbf{c}_{s}\right)=1$ if the source is outside the cylinders and is 0 when the source is inside one of the cylinders. The term $\chi_{l}^{\text {int }}\left(\mathbf{c}_{s}\right)=1$ when the source is inside cylinder $l$ and is 0 otherwise. By using Maxwell's equations we can calculate the components of the magnetic field $\mathbf{H}=\left(H_{x}, H_{y}\right)$, with 


$$
\mathbf{H}=\frac{\nabla \times \mathbf{E}}{i k Z_{0}},
$$

where $Z_{0}=\sqrt{\mu_{0} / \varepsilon_{0}}$ is the free space impedance.

The above method is highly accurate and numerically effective. As an illustration, in Fig. 2 we present the intensity distribution $I=\left|V^{e}\left(\mathbf{r} ; \mathbf{c}_{s}\right)\right|^{2}$ for a cluster of $N_{c}=317$ cylinders with the refractive indices $n_{l}$ uniformly distributed in the range $2.2-3.8$ and radii $a_{l}=0.4 d$ excited by a line source located at the center of the middle cylinder for one realization. Here $d$ is the distance between the centers of the neighboring cylinders. The cylinders form a finite cluster of approximately circular shape with cylinder centers located in a square lattice. The calculations have been done for a gap wavelength of the corresponding infinite structure, $\lambda / d=1.625$. Note the tendency of the field to concentrate at the center of the cluster, reflecting the localized nature of gap modes.

\section{TRANSPORT $l_{T}$ AND SCATTERING MEAN FREE PATH $l_{S}$ IN TWO-DIMENSIONAL RANDOM MEDIA}

In appropriate regimes, a suitable description of wave propagation in strongly scattering disordered media is the diffusion approximation, in which the wave equation for the fields is replaced by the simpler diffusion equation for the intensity. The main parameters involved in the diffusion approximation are the diffusion coefficient $D$, the transport velocity $v_{E}$, the transport mean free path $l_{t}$, and the scattering or elastic mean free path $l_{s}$. The transport velocity $v_{E}$ is considered in Sec. IV, while here we consider the properties of $l_{s}$ and $l_{t}$.

The scattering mean free path $l_{s}$ is the average distance between two successive scattering events, while the transport mean free path $l_{t}$ is the characteristic distance over which the direction of the momentum of photons becomes randomized. In the limit of low scatterer concentration, they can be calculated using

$$
\begin{aligned}
l_{s} & =\frac{1}{\rho \sigma_{t}}, \\
l_{t} & =\frac{l_{s}}{1-\left\langle\cos \theta_{s}\right\rangle},
\end{aligned}
$$

where $\rho$ is the concentration of the scatterers, $\theta_{s}$ is the scattering angle, and $\sigma_{t}$ is the total scattering cross section. This is $[29]$

$$
\sigma_{t}=\int_{0}^{2 \pi} \sigma_{d}(\theta) d \theta
$$

for two dimensional problems, where $\sigma_{d}(\theta)$ is the differential scattering cross section

$$
\sigma_{d}(\theta)=\lim _{r \rightarrow \infty} r \frac{\left|\mathbf{S}_{s}(\theta)\right|}{\left|\mathbf{S}_{i}\right|} .
$$

Here $\left|\mathbf{S}_{i}\right|$ is the Poynting vector of the incident plane wave and $\left|\mathbf{S}_{s}\right|$ is that of the plane wave scattered by a single inclusion, $\theta$ is the angle between the incident and scattered waves and $r$ is the distance from the center of the inclusion. For circular scatterers $\sigma_{d}$ and $\sigma_{t}$ can be calculated in closed form [29-31]. The Poynting vector of the scattered field $\left|\mathbf{S}_{s}\right|$ can be calculated by using the scattered part of the fields (3) and (4), giving

$$
\begin{aligned}
\sigma_{d}(\theta) & =\frac{2}{\pi k} \sum_{m=-\infty}^{\infty} \sum_{p=-\infty}^{\infty} B_{m} B_{p}^{*} e^{i(m-p) \theta e^{i(p-m) \pi / 2}}, \\
\sigma_{t} & =\frac{4}{k} \sum_{m=-\infty}^{\infty}\left|B_{m}\right|^{2}
\end{aligned}
$$

where

$$
B_{m}=\frac{n J_{m}^{\prime}(n k a) J_{m}(k a)-J_{m}(k n a) J_{m}^{\prime}(k a)}{n J_{m}^{\prime}(n k a) H_{m}^{(1)}(k a)-J_{m}(n k a) H_{m}^{(1)^{\prime}}(k a)}
$$

The average $\left\langle\cos \theta_{s}\right\rangle$ is 


$$
\left\langle\cos \theta_{s}\right\rangle=\frac{1}{2 \pi \sigma_{t}} \int_{0}^{2 \pi} \sigma_{d} \cos \theta d \theta=\frac{\operatorname{Re} \sum_{m=-\infty}^{\infty} B_{m} B_{m+1}^{*}}{\sum_{m=-\infty}^{\infty}\left|B_{m}\right|^{2}},
$$

where $n$ is the refractive index of the cylinder, the medium separating the cylinders is taken to have unit refractive index, $a$ is the radius and $k=2 \pi / \lambda$ is the wave number. Equations (5) and (6) hold only for low concentrations of scatterers with filling fraction $f \lesssim 0.1$ [32]. For higher concentrations of scatterers $0.1 \lesssim f \lesssim 0.6$ the scattering cross-section $\sigma_{t}$ must be rescaled as $\sigma_{t} \rightarrow \sigma_{t}(1-f)$ [29], while for the $f \gtrsim 0.6$ a more complicated rescaling parameter needs to be used [33].

In the diffusive propagation regime the relation

$$
\lambda / 2 \pi \ll l_{t} \ll L
$$

between the wavelength $\lambda$, the transport mean free path $l_{t}$ and the sample size $L$ of the medium must hold [28]. The lower limit in (13) is given by the Ioffe-Regel condition $k l_{t} \sim 1$ that determines the mobility edges of the Anderson transition for three-dimensional problems.

To illustrate the parameters that favor diffusive propagation we plot the averages, obtained from the equations of this section, $\left\langle l_{t}\right\rangle$ (solid line) and $\left\langle l_{s}\right\rangle$ (dashed line) as functions of the wavelength $\lambda$ in Fig. 3 for a cylinder with the refractive index uniformly distributed in the range $2.8<n_{c}<3.2$. The radii of the cylinders are $a=0.4 d$, where $d$ is the distance between the centers of two neighboring cylinders, and the filling fraction is $f=\pi a^{2} / d^{2} \approx 0.5$. Figure 3 shows that for long wavelengths $l_{t} \approx l_{s}$, while for shorter wavelengths, they are different. This is understandable, because for longer wavelengths the asymmetry parameter (12) approaches zero, since in this regime the monopole term $B_{0}$ is dominant in the cross section (9) and can make only a small contribution to the asymmetry parameter (because of the term $B_{m} B_{m+1}^{*}$ in 12). The horizontal and sloped straight lines indicate the predicted lower and upper bounds for diffusive propagation (13). The scattering mean free path $l_{s}$ also can be determined by the decay length of the average single-particle Green's function $\langle G\rangle[29]$. Due to randomness the effective refractive index of the medium renormalizes and acquires a complex part $k_{\mathrm{eff}}=k_{\mathrm{eff}}^{\prime}+i /\left(2 l_{s}\right)$. Here $k_{\mathrm{eff}}^{\prime}$ is the real part of the effective refractive index, which includes the real part of the forward scattering cross-section [29].

Figure 4 shows the dependence of $|\langle G\rangle|^{2}$ on the distance along the $y$ axis for the cluster of 317 cylinders with random refractive index uniformly distributed in the range $2.8-3.2$. The cluster is excited by a point source in the center of the central cylinder and the pass band wavelength is $\lambda / d=1.4$. The transport mean free path is equal to $l_{t} / d=3.29$ for this wavelength (see the solid line in Fig. 4). The slope of the straight lines on Fig. 4 determine the scattering mean free path $l_{s} / d=2.0 \pm 0.2$, which is consistent with (5) scaled by a factor $1-f$, which gives the value $\left\langle l_{s}\right\rangle / d=2.1$. This exponential decay is simply the decay of the coherent intensity $|\langle G\rangle|^{2} \approx e^{i 2 k_{\text {eff }} r} \approx e^{-r / l_{s}}[29]$.

Note that the results shown in Fig. 4, as in all later figures, have been calculated by averaging over an ensemble of 200 randomly chosen cluster realizations. This ensemble size seems sufficient to give stable results, even in the cases of strong randomization investigated.

\section{TRANSPORT VELOCITY IN TWO-DIMENSIONAL RANDOM MEDIA}

The speed of energy transport is one of the main characteristics of wave propagation in disordered media. In spite of its importance this quantity was only treated phenomenologically until recently [34,35]. In the low concentration limit a microscopic derivation of the speed of light is given in [12], while for the high concentration limit, different effective medium models have been developed [15,17]. All of these approaches, as models, have successes and shortcomings [18]. Therefore it is important to study this problem rigorously for some model problems that allow exact solution. The multipole method [20-25] is well adapted to the accurate calculation of the transport velocity from first principles.

The transport velocity $v_{E}$ is defined to be the ratio of the averaged energy flux determined from the Poynting vector $\mathbf{S}$ to the averaged energy density of the wave $W$ (where averaging is over a sufficiently large random ensemble)

$$
\mathbf{v}_{E}=\langle\mathbf{S}\rangle /\langle W\rangle \text {. }
$$

This definition was originally given by Brillouin [34] and correctly calculated by Loudon [35]. The group velocity $v_{g}=d \omega / d k$ does not take into account the multiple scattering of waves [12] over random distributions of scatterers. Furthermore, near resonances it can become greater than the speed of light in vacuum [36]. The calculation of the transport velocity given by (14) is consistent with experiments [12] and does not give values greater then speed of light. 
Before considering the speed of wave propagation in disordered photonic crystals it is first useful to calculate the transport velocity of waves in the ordered case. The geometry of the cluster is the same as in Fig. 2 . The refractive indices of the cylinders are $n_{c}=3$. The line source is located in the center of the middle cylinder. The solid line in Fig. 5 represents $\left|\mathbf{v}_{E}\right| / c$ from (14) versus the position $y$ with the source located in the middle of the central cylinder for the wavelength $\lambda / d=1.625$, which is located just inside the gap region of the corresponding infinite structure. It is seen that the transport velocity can be as low as $10^{-8} c$, for a cluster of this size. The linear trends indicate that the transport velocity increases exponentially away from the center of the cluster. The transport velocity $v_{E}$ tends to zero in the vicinity of the source, as a consequence of symmetry (the absence of a preferred direction for this vector quantity). It is also seen that just outside the cluster at $y / d= \pm 11$ the transport velocity $v_{E} / c$ reduces its value to 0.3 before it rapidly approaches the free space value away from the edge of the cluster. This local minimum of $v_{E}$ is due to a strong interference effect at the edge of the cluster. We calculated $v_{E} / c$ also for a bigger cluster with $N_{c}=625$ cylinders and this reduction of the transport velocity $v_{E} / c$ outside the cluster still takes place.

The dashed line on Fig. 5 is $v_{E} / c$ versus position for the wave with the pass band wavelength $\lambda / d=3.425$. For this wavelength the transport velocity oscillates around its homogeneous value $v_{E} / c=1 / n_{\text {eff }}=0.44$ calculated using the linear mixing formula $\varepsilon_{\text {eff }}=1-f+f\left\langle\varepsilon_{l}\right\rangle$ [24], which is rigorous for this polarization, and approaches the free space value $c$ outside the cluster.

Figure 6 shows the same relationship as in Fig. 5 but for a disordered photonic crystal with uniformly distributed cylinder refractive index in the range $2.9<n_{c}<3.1$ (dashed line, middle curve, weak disorder) and in the range $2.2<n_{c}<3.8$ (dotted line, top curve, strong disorder) averaged over 200 realizations. The solid line is $v_{E} / c$ for a fully ordered photonic crystal with $n_{c}=3.0$. For weak disorder $2.9<n_{c}<3.1, v_{E} / c \approx 10^{-5}$ at the center of the cluster, while strong disorder has a larger effect on the transport velocity. Note that although the transport velocity $v_{E} / c$ is substantially increased for strong disorder compared to $v_{E} / c$ for the perfect crystal, its value is nearly 15 times less than the free space value. Similar low values of the transport velocity have been reported experimentally $[12,37]$. As we approach the edge of the cluster the transport velocity $v_{E} / c$ rapidly approaches the free space value.

\section{DIFFUSION, ANOMALOUS DIFFUSION AND INCIPIENT ANDERSON LOCALIZATION}

In this section we consider the different regimes of wave propagation: the diffusive and anomalous diffusive regimes, and evidence for incipient Anderson localization. The transport mean free path $l_{t}$ and velocity $v_{E}$ are important characteristics of diffusive propagation. The diffusion constant $D$ is given by $D=v_{E} l_{t} / 2$ for two dimensional problems. In the diffusion approximation, where the transport mean free path is bigger than the wavelength, the wave equation for the electric field component (1) can be replaced by a diffusion equation for the wave intensity $[29,30]$. For monochromatic problems with a source at $c_{s}$ the diffusion equation takes the form

$$
\nabla^{2} I=-\frac{2 P_{0}}{l_{t}} \delta\left(\mathbf{r}-\mathbf{c}_{s}\right)
$$

where $P_{0}$ is the total emitted energy, $l_{t}$ is the transport mean free path, and $I$ is the intensity given by $I=\left\langle G G^{*}\right\rangle-$ $|\langle G\rangle|^{2}[29]$. Note that the coherent intensity $|\langle G\rangle|^{2}$ is negligible here (for sufficient degree of disorder) and can be disregarded in (15). For example for the cluster with 317 cylinders and the refractive index disorder in which the refractive index is uniformly distributed in the range $n_{l} \pm Q$ the degree of disorder $Q=0.2$ is sufficient to render negligible the coherent intensity.

The diffusion equation is usually solved with boundary conditions [29] at the edge of the sample

$$
I \pm \frac{2 l_{t}}{3} \frac{\partial I}{\partial \nu}=0
$$

where $\nu$ is the unit outward normal from the diffusive region. Note that the diffusion equation as well as the proposed boundary conditions (16) are approximate [29,30]. Other boundary conditions are discussed in [38]. These boundary conditions are obtained by the requirement that the intensity flux directed inward from the exterior of the disordered sample vanishes. Here, we first solve the exact Helmholtz equation using the multipole method and find the averaged intensity value at the edge of the cluster $r=L$. Then we use this value as a boundary condition for the diffusion equation (15). By using this boundary condition we automatically satisfy the requirement of the absence of the inward flux. Given this boundary condition the analytical solution of the diffusion equation (15) inside the cluster takes the form

$$
I=-\frac{P_{0}}{\pi l_{t}} \ln \frac{\left|\mathbf{r}-\mathbf{c}_{s}\right|}{L}+I_{0},
$$


where $l_{t}$ is the transport mean free path, $I_{0}$ is the exact value for the intensity at the edge of the cluster found from the numerical solution of the Helmholtz equation and $L$ is the size of the cluster, which we take to have a circular shape. For points outside the cluster $\left|\mathbf{r}-\mathbf{r}_{s}\right|>L$ we continue the diffusive solution (17) by the relation

$$
I=I_{0} \frac{L}{r}
$$

where $I_{0}$ is the intensity at the edge of the cluster, $L$ is the size of the cluster and $r$ is the distance from the source. Note that for two-dimensional problems the intensity of a point source located at the origin decays as $1 / r$ far from the source.

Below we consider both the transport properties of a wave with pass band and gap wavelengths of the corresponding regular infinite photonic crystal.

\section{A. Diffusion and anomalous diffusion for a pass band wavelength}

In Fig. 7 we plot $I$ versus $y$ for a source located at the middle of the central cylinder for a pass wavelength $\lambda / d=1.4$ (indicated by the letter $A$ on Fig. 3) of a corresponding infinite structure and for a cluster as in Fig. 2 . The refractive indices $n_{l}$ of cylinders are uniformly distributed in the range $2.8-3.2$. The oscillating line is the exact solution of the Helmholtz equation averaged over $N_{r}=200$ random realizations and the dashed line is the analytical solution given by (17) for points inside the cluster and by (18) for points outside the cluster. There is good agreement between two curves. Therefore, we conclude that this is the diffusive regime of wave propagation.

In Fig. 8 we plot the same relation as in Fig. 7, but for stronger disorder, where the refractive index is uniformly distributed in the range $2.2-3.8$. The dashed line is the solution (17) of the diffusion equation for points inside the cluster and the relation (18) for points outside. In order to obtain the fit for this case we had to rescale the transport mean free path from the value $l_{t} \approx 3.29$ indicated by the letter $A$ in Fig. 3 ( corresponding to the Ioffe-Regel value $k l_{t} \approx 14.77$ ) to the value $l_{t} / d \approx 0.55 \pm 0.15$ indicated by the letter $A^{*}$ in Fig. 3 (corresponding to the Ioffe-Regel value $\left.k l_{t} \approx 2.47\right)$. This reduction of the transport mean free path is an indication of the anomalous diffusion regime. Note that for the Anderson transition to take place the condition $k l_{t} \approx 1$ must be satisfied, corresponding to the transport mean free path $l_{t} \approx 0.22$. To achieve this regime, bigger clusters or/and possibly stronger disorder are required, requiring computer times which are prohibitive at present.

\section{B. Diffusion and anomalous diffusion for a gap wavelength}

In Fig. 9 we plot $\left\langle|G|^{2}\right\rangle$ versus $y$ for the gap wavelength $\lambda / d=1.625$ (indicated by the letter $B$ on Fig. 3 ) which is located just inside the edge of the gap for the corresponding infinite structure. It has been suggested [39] that photons with gap wavelengths are easier to localize by the Anderson mechanism. The refractive indices of the cylinders are uniformly distributed in the range $2.2-3.8$ (top curve), $2.8-3.2$ (middle curve) and regular $n_{l}=3$ for the bottom curve. Here we investigate transition from gap localization (bottom curve) to possible Anderson localization (top curve) as we increase the degree of disorder. It might be expected that we would first see the classical diffusive regime of wave propagation as we increase the disorder. However, it turns out that for a gap wavelength this transition takes place directly (or at least, very rapidly) to the anomalous diffusive regime. The dotted line on the oscillating top curve for points inside the cluster is the solution of the diffusion equation (15) with the rescaled transport mean free path $l_{t}^{*}$. We obtain the fit for the value $l_{t}^{*} / d=0.3$ indicated by the letter $B^{*}$ in Fig. 3 , which corresponds to the value of the Ioffe-Regel number $k l_{t}^{*} \approx 1.16$, close to the edge of the Anderson transition $k l_{t}^{*} \approx 1$. Interestingly, the exponential relationship $f(x)=0.25 * \times \exp (-|x|) / 3$ is also a good fit for the intensity curve and one can estimate the localization length $l / d \approx 3$.

The above calculations suggest that for the gap wavelengths the transition from gap localization to Anderson localization takes place through the anomalous diffusive regime, in which the transport mean free path can no longer be taken as constant, but is a function of the size of the cluster and the degree of the disorder.

\section{CONCLUSION}

We have investigated the different transport regimes: regular/ordered, diffusive, and anomalous diffusive regimes of wave propagation in disordered photonic crystals for both gap and pass band wavelengths of corresponding infinite 
regular structures. The main parameters of diffusive propagation: the transport velocity $v_{E}$, and the transport and scattering mean free paths, $l_{t}$ and $l_{s}$, have been calculated. The parameters that corresponds to diffusive propagation have been quantified.

For the first time the transport velocity $v_{E} / c$ introduced by Brillouin has been calculated from first principles for a random medium with an intermediate filling fraction $f=0.5$. It was found that the transport velocity of light $v_{E}$ in ordered finite-size photonic crystals can be substantially lower $\left(v_{E} / c \approx 10^{-8}\right)$ than the free space value for a gap wavelength. The effects of the disorder on the transport velocity were also considered and it has been shown that $v_{E} / c$ can be more then 15 times less than in free space, which is in agreement with the experimental results reported earlier. Although the transport velocity is rather sensitive to the degree of disorder, use of bigger clusters can substantially reduce the transport velocity even for weak disorder. The calculation of the transport velocity $v_{E}$ based on our rigorous method can serve as a basis on which the accuracy and the limits of applicability of different effective medium models can be verified and checked.

For a wavelength in the pass band and a wavelength in the gap we have calculated the transport properties of waves. In Fig. 10 we schematically illustrate and summarize the overall results obtained. Wave propagation for a pass band wavelength of the corresponding infinite cluster for weak disorder is diffusive (region $D$ in Fig. 10), while for strong disorder the transport becomes anomalously diffusive (region $A D$ in Fig. 10). To demonstrate the Anderson transition bigger clusters and/or larger disorder are needed. For a gap wavelength we have investigated the transition from gap localization to Anderson localization and have shown that this transition takes place directly through the anomalous diffusive regime (region $A D$ in Fig. 10) and the threshold of the Anderson transition is more easily achieved (region $A L$ in Fig. 10) [39]. An incipient Anderson transition was demonstrated. This qualitative smooth picture of the Anderson transition in $2 D$ is in agreement with the scaling theory of localization.

\section{ACKNOWLEDGMENTS}

This work was supported by the Australian Research Council.

[1] C.M. Soukoulis, Photonic Crystals and light localization in the 21st century (Kluwer, Dordrecht, 2001).

[2] S. John, Phys. Rev. Lett. 58, 2486 (1987).

[3] E. Yablonovitch, Phys. Rev. Lett. 58, 2059 (1987).

[4] Photonic Band Gap Materials, Edit. C.M. Soukoulis, NATO Series Vol. 315, (Kluwer, Dordrecht, 1995).

[5] J.D. Joanopoulos, R.D. Meade, and J.N. Winn, Photonic Crystals: Molding the Flow of Light (Princeton University, New Jersey, 1995).

[6] P. Sheng, Introduction to Wave Scattering, Localization, and Mesoscopic Phenomena (Academic, San Diego, 1995).

[7] E. Abrahams, P.A.Anderson, D.C. Licciardello, and T.V. Ramakrishnan, Phys. Rev. Lett. 42, 673 (1979).

[8] Scattering and localization of classical waves in random media, Edt. P. Sheng (World Scientific, Singapore, 1990).

[9] A.R. McGurn, P. Sheng, and A.A. Maradudin, Opt. Commun. 91, 175 (1992).

[10] H. De. Raedt, A. Lagendijk, and P. de Vries, Phys. Rev. Lett. 62, 47 (1989).

[11] D. Felbacq, D. Maystre, and G. Tayeb, J. Mod. Opt. 42, 473 (1995).

[12] M.P. Albada, B. A. van Tiggelen, A.Lagendijk, and A.Tip, Phys. Rev. Lett. 66, 3132 (1991).

[13] B. A. van Tiggelen, A.Lagendijk, M.P. Albada, and A.Tip, Phys. Rev. B 45, 12233 (1992).

[14] C.M. Soukulis, S. Datta, and E.N. Economou, Phys. Rev. B 49, 3800 (1994).

[15] H.P. Schriemer, M.L. Cowan, J.H. Page, P. Sheng, Zhengyou Liu, and D.A. Weitz, Phys. Rev. Lett. 79, 3166 (1997).

[16] K.Busch, C.M. Soukoulis, E.N. Economou, Phys. Rev. B 52, 10834 (1995).

[17] K.Busch, and C.M. Soukoulis Phys. Rev. Lett. 75, 3442 (1995).

[18] C.M. Soukoulis, K. Busch, M. Kafesaki Phys. Rev. Lett. 82, 2000 (1999).

[19] H.P. Schriemer, M.L. Cowan, J.H. Page,Ping Sheng, Zhengyou Liu, and D.A. Weitz, Phys. Rev. Lett. 82, 2001 (1999).

[20] D.Felbacq, G.Tayeb, and D.Maystre, JOSA A, 11, 2526 (1994).

[21] G.Tayeb, and D.Maystre, J. Opt. Soc. Am. A 14, 3323 (1997).

[22] L.-M. Li, Z.-Q. Zhang, Phys. Rev. B 58, 9587 (1998).

[23] E. Hoskinson and Z. Ye, Phys. Rev. Lett. 83, 2734 (1999).

[24] A.A. Asatryan, P.A. Robinson, L.C. Botten, R.C. McPhedran, N.A. Nicorovici, C.Martijn de Sterke, Phys. Rev E 60, 6118 (1999). 
[25] L.C. Botten, N.A. Nicorovici, A.A.Asatryan, R.C. McPhedran, C. Martijn de Sterke, P.A. Robinson, J. Opt. Soc. Am A 17, 2165 (2000).

[26] A.A. Asatryan, K. Busch, R.C. McPhedran, L.C. Botten, C. Martijn de Sterke, N.A. Nicorovici, Phys. Rev. E 63, 046612 (2001).

[27] A.A. Asatryan, S.Fabre, K. Busch, R.C. McPhedran, L.C. Botten, C. Martijn de Sterke, N.A. Nicorovici, Optics Express 8, 191 (2001).

[28] E. Amic, J.M. Luck, and Th. M. Nieuwenhuizen, J. Phys. A 29, 4915 (1996).

D.A. Weitz, and

[29] A. Ishimaru, Wave Propagation and Scattering in Random Media v.1, v.2 (Academic, NY, 1978).

[30] L. A. Apresyan, Yu.A. Kravtsov Radiation Transfer Statistical and Wave Aspects (Gordon and Breach, The Netherlands, 1996).

[31] Van de Hulst, H.C. Light scattering by small particles, (Wiley, NY, 1957).

[32] K. Busch, C.M. Soukoulis and E.N. Economou, Phys. Rev. B 50, 93 (1994).

[33] C.I. Beard, T.H. Kays, and V. Twersky, IEEE Trans. on Antennas and Propagation 15, 99 (1967).

[34] L. Brillouin, Wave propagation and Group Velocity, (Academic, New York, 1960).

[35] R. Loudon, J. Phys. A 3, 233 (1970).

[36] M. Born, and E. Wolf, Principles of Optics (Pergamon, Oxford, 1984).

[37] M. Notomi, K. Yamada, A. Shinya, J. Takahashi, C. Takahashi, and I. Yokohama, Phys. Rev. Lett. 87 253902, (2001).

[38] R.C. Haskell, L.O. Svaasand, T.-T. Tsay,T.-C. Feng, and M.S. McAdams, JOSA A 11, 2727 (1994).

[39] S. John, Comm. Condense Matter Physics, 14, p.193, (1988) 


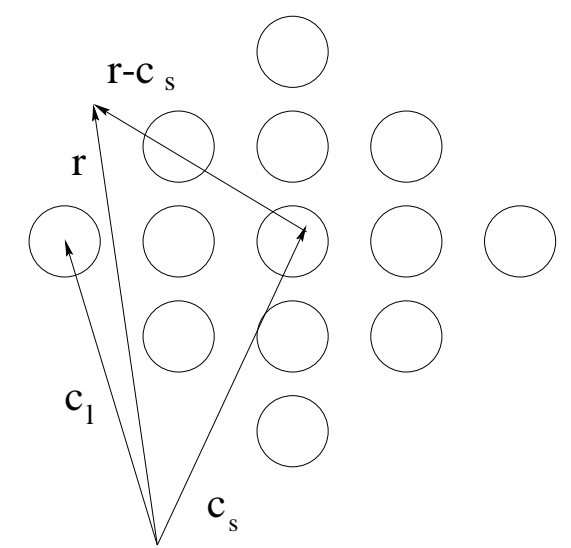

FIG. 1. The geometry of the problem. The positions of the centers of the cylinders are given by the vectors $\mathbf{c}_{l}$ and that of the source by $\mathbf{c}_{s}$, while $\mathbf{r}$ is the position at which the Green function is calculated .

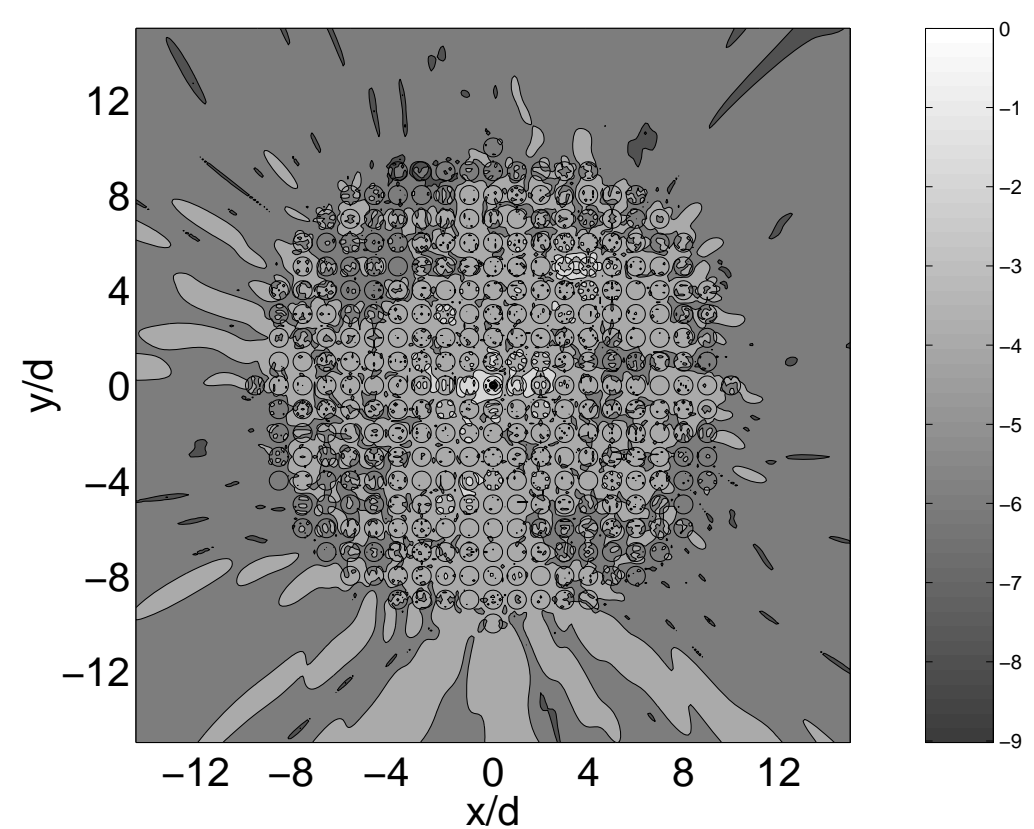

FIG. 2. Intensity distribution for a source at the center of the central cylinder of a cluster with $N_{c}=317$ cylinders. The black circles indicate the positions of the cylinders. The refractive indices of the cylinders are random with a uniform distribution over the range $2.2-3.8$. 


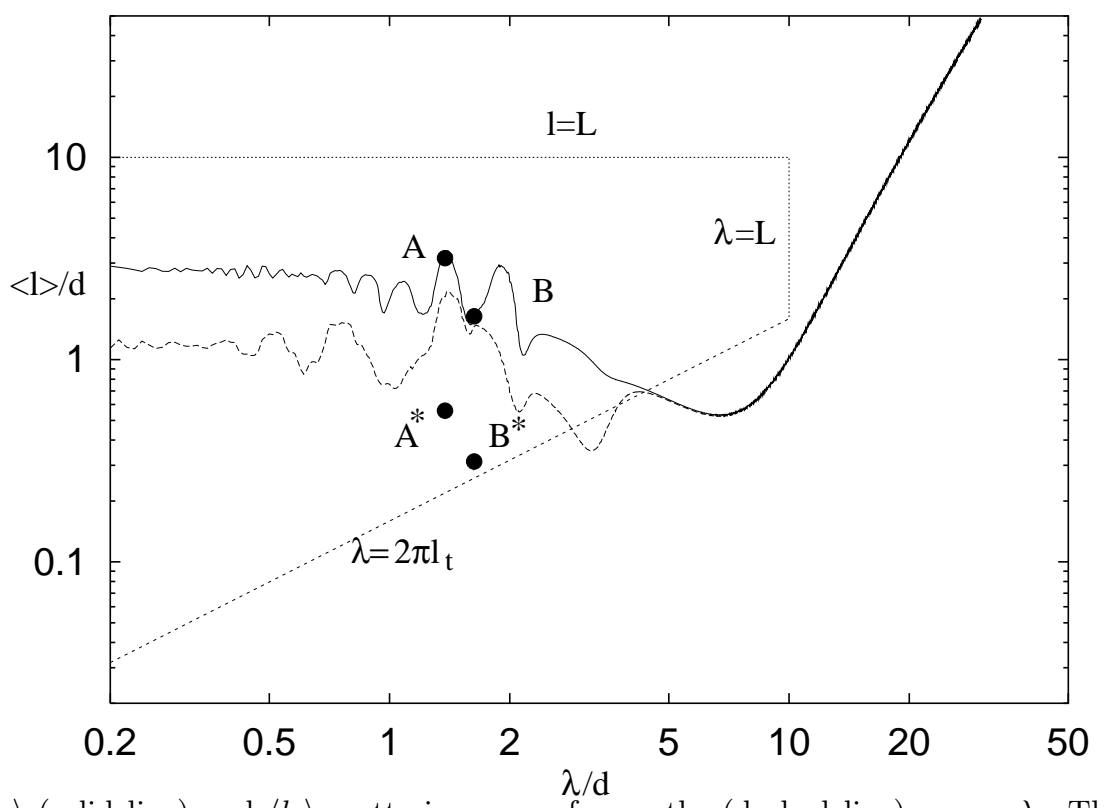

FIG. 3. Transport $\left\langle l_{t}\right\rangle$ (solid line) and $\left\langle l_{s}\right\rangle$ scattering mean free paths (dashed line) versus $\lambda$. The region formed by the straight lines is the region that satisfies the condition (13) for diffusive propagation. The sloped straight line is the limiting value of the Ioffe-Regel criterium $\lambda=2 \pi l_{t}$ from below, while the horizontal and vertical straight lines are the limitations due to the size of the cluster $L / d=10$. The black dots indicated by letters $A, A^{*}, B$, and $B^{*}$ are discussed in Sec. V

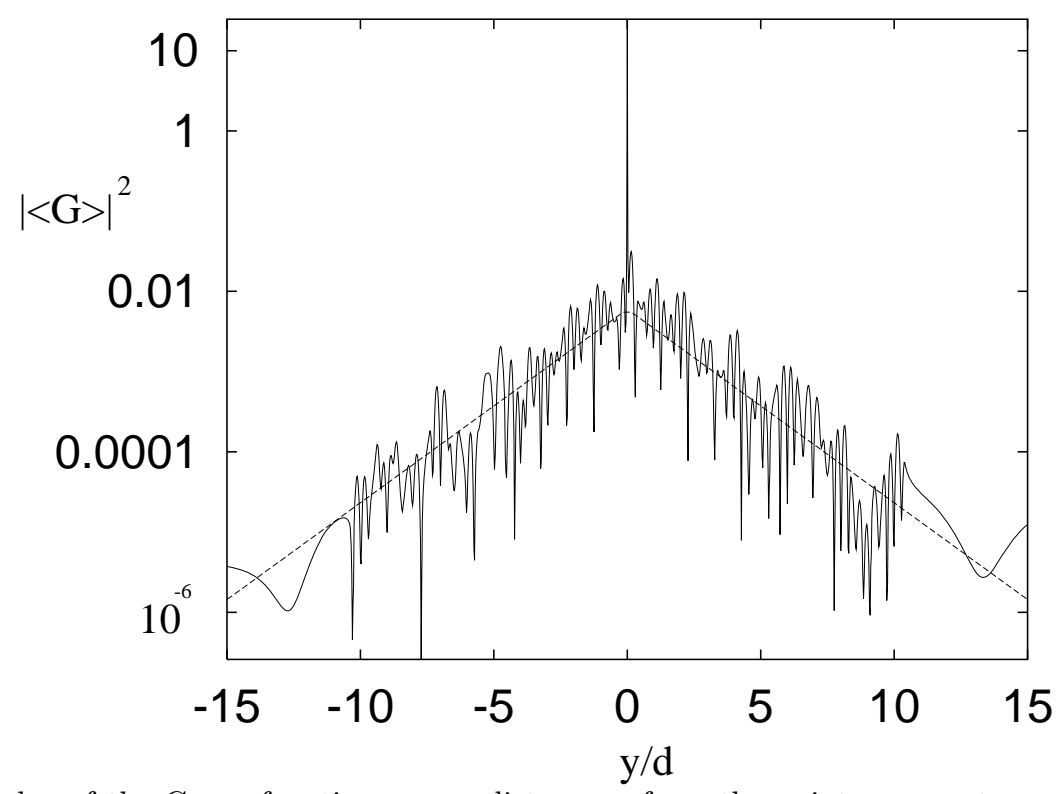

FIG. 4. Average modulus of the Green function versus distance $y$ from the point source at $x_{s}=0, y_{s}=0$. The slope of the straight lines determines the scattering mean free path $l_{s}$ (see text), which is in agreement with Eq. (5) scaled by a factor $1-f$. 


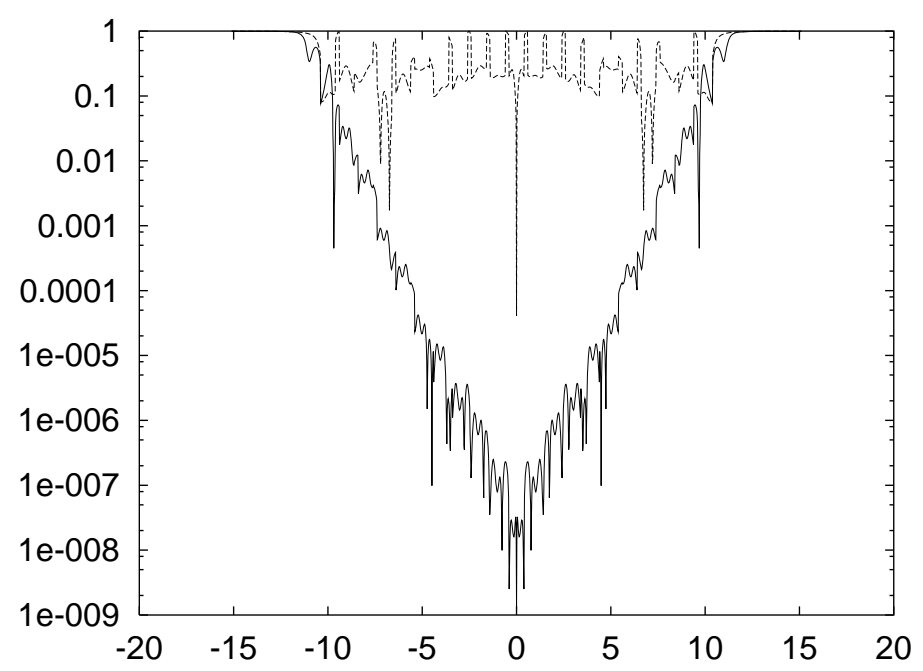

FIG. 5. Transport velocity $v_{E} / c$ for an ordered photonic crystal versus the distance from the line source at the center of the central cylinder at $x_{s}=y_{s}=0$; (solid line) for gap wavelength $\lambda / d=1.625$, and (dashed line) for pass band wavelength $\lambda / d=3.425$.

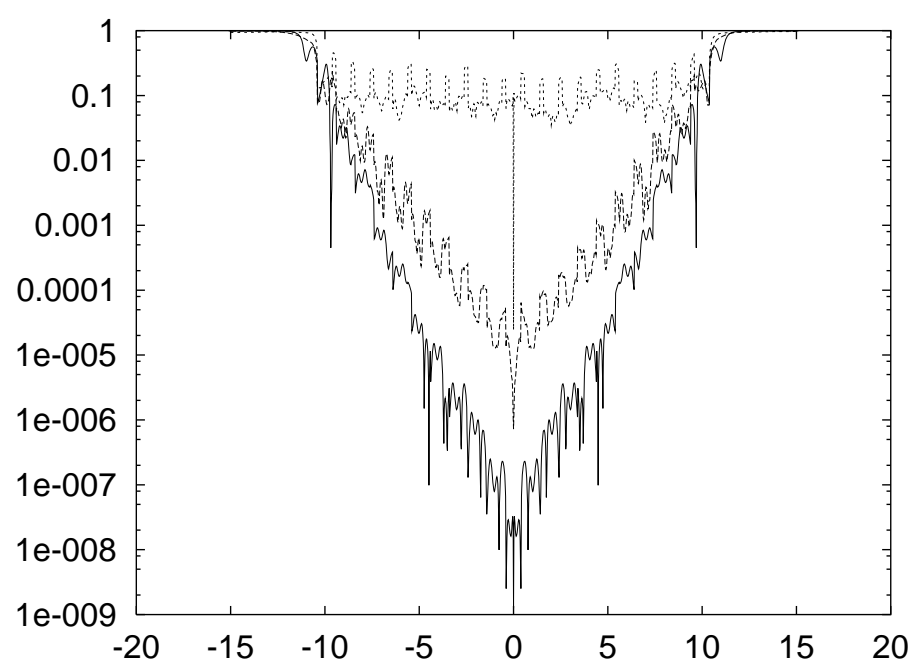

FIG. 6. The modulus of the transport velocity $v_{E} / c$ versus the distance from the line source at the center of the central cylinder at $x_{s}=y_{s}=0$ for an ordered photonic crystal (solid line) and disordered photonic crystal for a gap wavelength $\lambda / d=1.625$. The refractive index is distributed in the range $2.9-3.1$ (dashed line) and in the range $2.2-3.8$ for dotted line. 


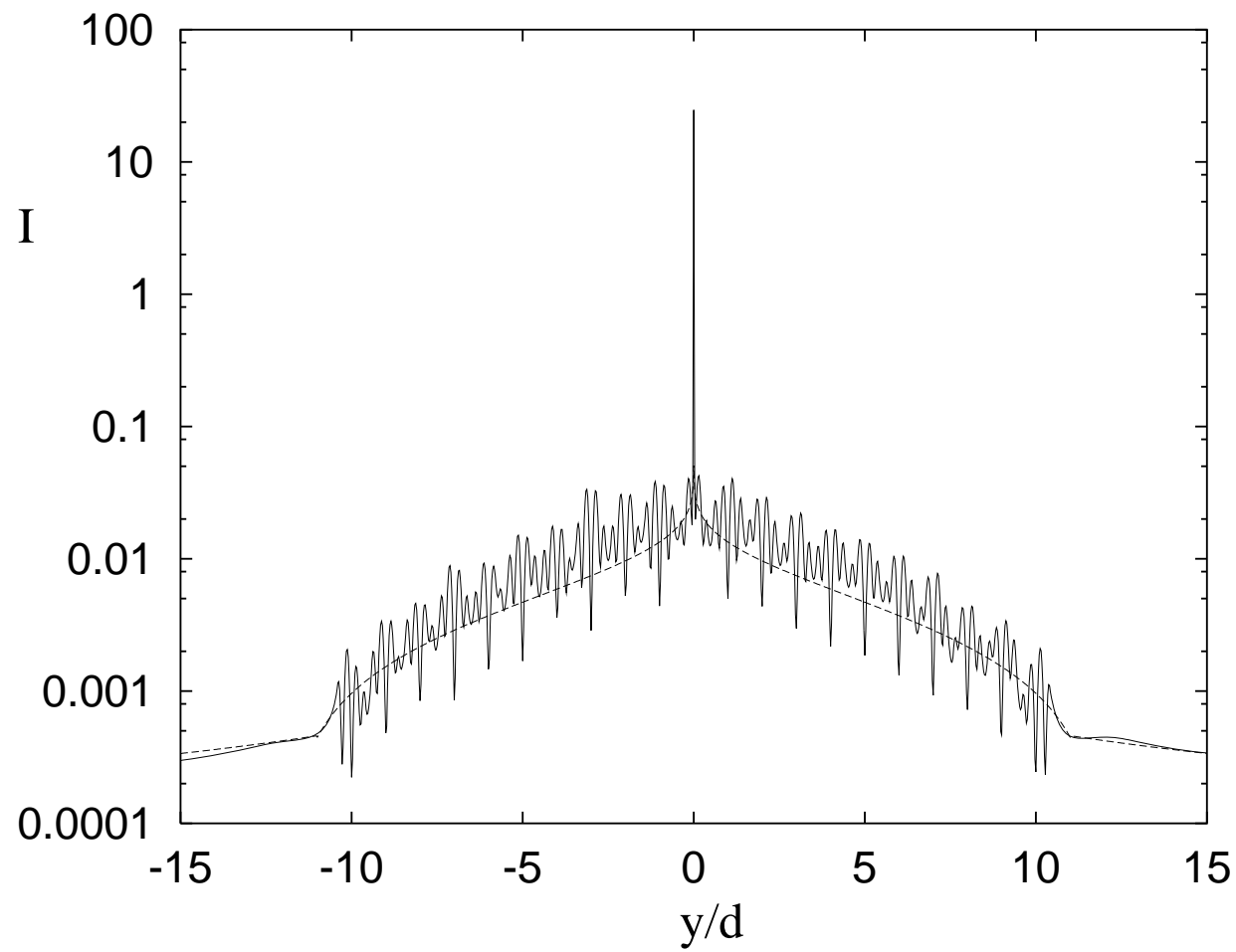

FIG. 7. Intensity versus distance from a point source at the center of the central cylinder at $x_{s}=y_{s}=0$; for a wavelength $\lambda / d=1.4$. The solid oscillating line is the averaged solution of the Helmholtz equation and the dashed line is the Eq. (15) for points inside of the cluster and Eq. (18) for points outside. The cluster comprises 317 cylinders and the refractive index distribution is uniform in the range 2.8-3.2.

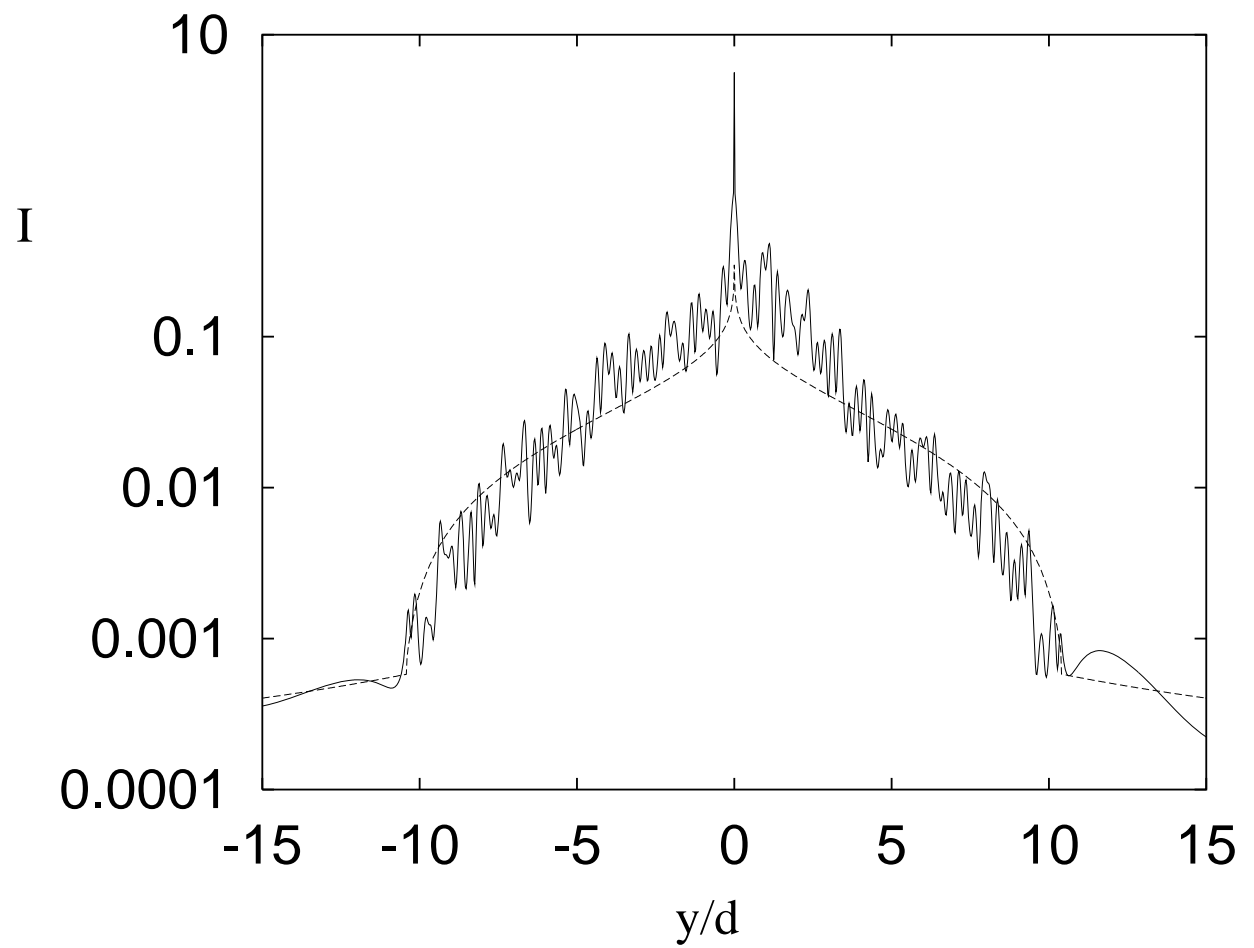

FIG. 8. The same relationship as in Fig. 7, but for the refractive index distribution in the range 2.2-3.8. Due to strong randomness the transport mean free path renormalizes from the value $\left\langle l_{t}\right\rangle / d \approx 3.29$ to the value $\left\langle l_{t}\right\rangle \approx 0.55$. This is the regime of the anomalous diffusion. 


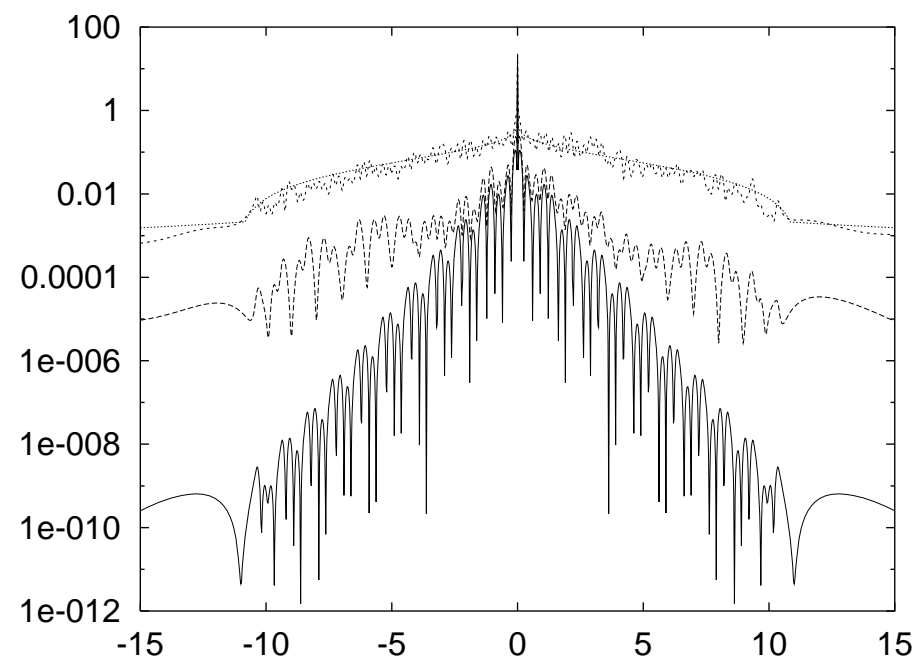

FIG. 9. Intensity $I$ versus the distance from a point source at the center of the central cylinder at $x_{s}=y_{s}=0$; for a gap wavelength $\lambda / d=1.625$. The transition from gap localization to Anderson localization. The refractive index of the cylinders are uniformly distributed in the range $2.2-3.8$ (top curve), and $2.8-3.2$ (middle curve), and have fixed $n_{l}=3$ for the bottom curve. The dotted line overlaying the oscillating top curve is the anomalous diffusion fit.

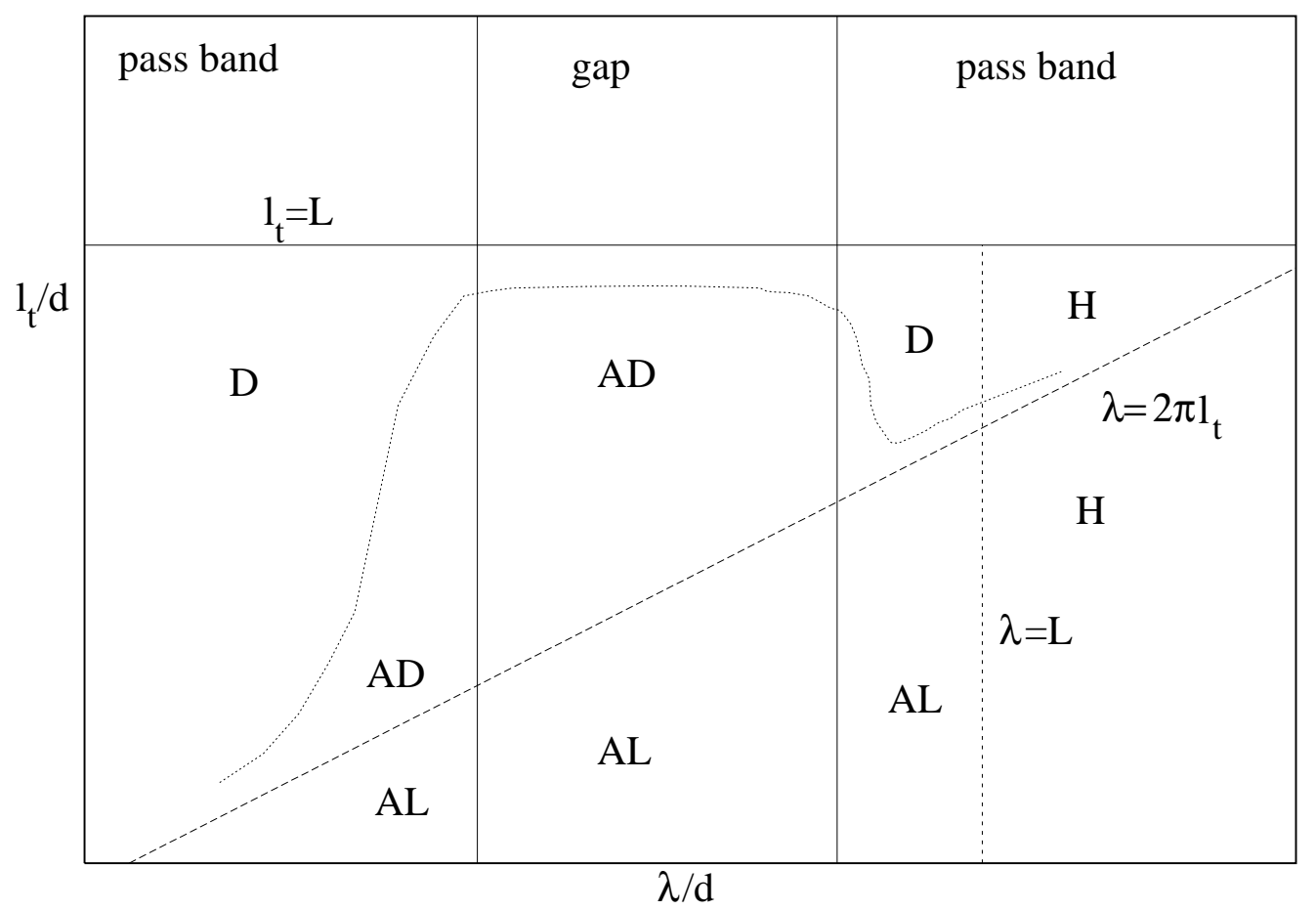

FIG. 10. Schematic picture of the different regimes of the wave propagation. $D$-stands for diffusion, $A D$-for anomalous diffusion, $A L$-Anderson localization, $H$-homogenization. The sloping line is an indication of the Ioffe-Regel criterion, while the dotted line gives an indication of the boundary between the normal and anomalous diffusion regimes. Reading from left, the vertical lines mark the edges of the bandgap and the size of the sample. 